\title{
A generalized model of active media with a set of interacting pacemakers: Application to the heart beat analysis
}

\author{
SERGEI RYBALKO ${ }^{\dagger}$ and EKATERINA ZHUCHKOVA
}

\author{
$\dagger$ Department of Physics, Kyoto University, Kyoto 606-8502, Japan \\ $\ddagger$ Physics Faculty, Moscow State University, Leninskie gory, Moscow 119992, Russia \\ E-mail: rybalko@polly.phys.msu.ru
}

\begin{abstract}
We propose a quite general model of active media by consideration of the interaction between pacemakers via their phase response curves. This model describes a network of pulse oscillators coupled by their response to the internal depolarization of mutual stimulations.

First, a macroscopic level corresponding to an arbitrary large number of oscillatory elements coupled globally is considered. As a specific and important case of the proposed model, the bidirectional interaction of two cardiac nodes is described. This case is generalized by means of an additional pacemaker, which can be expounded as an external stimulater. The behavior of such a system is analyzed. Second, the microscopic level corresponding to the representation of cardiac nodes by one- and two-dimensional lattices of pulse oscillators coupled via the nearest neighbors is described. The model is a universal one in the sense that on its basis one can easily construct discrete distributed media of active elements, which interact via phase response curves.
\end{abstract}

PACS 05.45.Ac, 82.40.Bj, 95.10.Fh, 87.19.Hh

Keywords: Active media; entrainment; cardiac dynamics; phase response curve.

Short title: A generalized model of active media 


\section{Introduction}

Representation of an active distributed system by ensembles of coupled excitable or oscillatory elements is very useful method of the analysis because it allows to understand main dynamical processes inherent in the considered medium. As is known, this approach goes back to the model of Wiener and Rosenblueth [Wiener \& Rosenblueth, 1946], according to which a medium consists of single elements being in one of three possible states: excited, refractory or rest. Later such models as coupled limit cycle oscillators and chaotic maps [Kaneko, 1990; Shibata \& Kaneko, 1998] have played an important role not only in a quite realistic description of active media but also in the understanding of a possible behavior of systems far from equilibrium. Many useful concepts like phase-locked patterns, synchronization and spatio-temporal chaos have become popular due to detailed studies of similar nonlinear models [Kuramoto, 1984; Kuramoto, 1995; Winfree, 2000].

Investigations of such an example of an active medium as cardiac tissue are of significant scientific interest owing to vital importance of its rhythm stability. Real heart cells exhibit oscillatory properties (can be reset and entrained), they are excitable and have a refractory time, during which they do not respond to external stimulation. Hence, the heart can be considered as consisting of oscillatory (conductive cardiomyocytes, which have automaticity) and excitable (contractile heart cells, which do not initiate electrical activity under normal conditions) elements.

Due to extraordinary complexity of the heart, many qualitative discrete and continuous models have been tested. Majority of computational models of cardiac tissue of last generation takes into consideration the kinetics of excitable cells, how the excitation propagates from cell to cell and how contractile cardiomyocytes are arranged and connected in space [Clayton, 2001]. Such models mainly serve for studying sustained by re-entrant activity lethal arrhythmia - ventricular fibrillation, during which the spatio-temporal behavior is very complex [Clayton et al., 2006].

Other models treat the cardiac tissue as an active conductive system, taking into account oscillatory properties of heart cells. In this case the cardiac rhythms can be described on the basis of the dynamical system theory (see e.g. [Courtemanche et al., 1989; Goldberger, 1990; Bub \& Glass, 1994; Glass et al., 2002; Loskutov et al., 2004] and refs. therein). 
Hereinafter we hold this approach.

Under normal conditions the electrical activity of the heart (action potentials) is spontaneously initiated in a region of the right atrium, sino-atrial (SA) node, so-called leading pacemaker. Automatic excitation is a distinctive feature not only of the cells of the SA node, but also of other conductive heart cells, so-called latent pacemakers. In addition, contractile cardiomyocytes can initiate a spontaneous action potential in pathology. Electrophysiological studies have suggested that the activity of cardiomyocytes with automaticity (e.g. P-cells of the SA node, of the atrioventricular (AV) junction, Purkinje cells) can be modulated by current pulses stimulating (super-threshold depolarizing) applied extracellularly [Jalife \& Moe, 1976; Sano et al., 1978; Jalife et al., 1980; Antzelevitch et al., 1982].

Effects of external stimuli on biological oscillators are observed in a wide range of species. Experimentally obtained characteristics can be represented by a phase response curve (PRC) [Jalife \& Moe, 1976; Antzelevitch et al., 1982; Reiner \& Antzelevitch, 1985]. To establish the shape of the PRC experimentally, stimulation of an oscillator at various phases of its intrinsic cycle is applied [Jalife et al., 1980; Guevara \& Shrier, 1987]. It has been found that in different pacemaker cells early stimuli delay the next pacemaker discharge and late pulses advance it. Therefore, the typical PRC shape is biphasic [Jalife et al., 1980; Reiner \& Antzelevitch, 1985].

The rhythm of autonomous biological oscillators can also undergo an external periodic perturbation (e.g. activity of cells of the AV junction is subjected to sinus rhythm), depending on both the stimulus magnitude and its phase within the cycle. It is known that when the frequency and the amplitude of the external periodic stimulation are varied, a diversity of phase diagrams can be established between the stimulus and the self-sustained oscillator (see e.g. [Loskutov et al., 2004]). In some situations the rhythm of the biological oscillator is entrained (or phase-locked) to the external stimulation so that for each $M$ cycles of the stimulation there are $N$ cycles of the autonomous oscillator rhythm. This occurs at a fixed phase (or phases) of the stimulus and is called $M: N$ phase-locking or entrainment, which appears as a time-periodic sequence. In particular, entrainment of $1: 1$, during which the rhythms of the oscillator and external stimulus are matched, is defined as synchronization phenomenon. 
In the present paper we develop a general simplified model describing a network of pulse oscillators coupled by their response to the internal depolarization of mutual stimulations. Our primary aim is to keep the model as simple as possible and to introduce a minimal number of parameters. Therefore, in our model the pacemakers are fully characterized by their intrinsic cycle length and are represented as pulse oscillators. Their interaction is described by PRCs. At first, we will consider two bidirectionally interacting pacemakers to

demonstrate the basic concepts of the model. Then we will apply this approach to construct a pacemaker network model with global coupling. As the following step, we will analyze two specific cases of this PRC based model of coupled pulse oscillators: two and then three interacting cardiac nodes. An additional pacemaker can also be expounded as an external stimulater. Our further intention is to go on to the next (microscopic) level and represent each pacemaker as an ensemble of interacting oscillatory elements. Extrapolation of our approach to one- and two-dimensional matrixes (or lattices) of pacemaker cells interacting via nearest neighbors concludes the present study.

\section{Development of the General Model}

In this part we construct a system with two pacemakers and then consider a quite general model of a set of interacting pacemakers coupled by their PRCs.

\subsection{Two interacting pacemakers: outline of the approach}

Consider two interacting pulse oscillators (or pacemakers) $A$ and $B$ with intrinsic periods of their autonomous beating $T_{a}$ and $T_{b}$ respectively. An interaction between oscillators is governed by so-called phase response curve (PRC). This means that a phase shift of one of the oscillators happens as a result of an impact of the another one. To construct an adequate mathematical model, it is necessary to accept some restrictions concerning the character of the interaction. We describe them briefly [Ikeda, 1982; Glass et al., 1986; Glass \& Zeng, 1990].

1. The phase of the disturbed oscillator is shifted to a new value instantly after an impact.

2. The phase shift depends only on two main parameters: a) on the phase difference 
of oscillators and b) on the influence strength. In turn, this influence strength depends on its amplitude and the coupling coefficient of the oscillators. In a real system the coupling coefficient is the average factor that shows how the strength of the pulse decreases during its passing from one oscillator to another. Thus, the phase shift $\Phi$ determining a new phase of the disturbed oscillator with period $T$ can be represented as follows:

$$
\Phi=\Delta / T \equiv \Phi(\varphi, \varepsilon)
$$

where $\Delta$ is the time shift of the disturbed oscillator, $\varphi$ is the phase difference of the oscillators and $\varepsilon$ is the influence strength.

Pacemakers can be represented as a set of separated firing peaks on a time scale. Assume that the instants of the last firings of the oscillators $A$ and $B$ are $a$ and $b$ respectively (Fig. 11). Note that $a$ and $b$ are the moments of the impacts after all previous phase shifts of the oscillators. In other words, one can observe the oscillators' firings at these particular instants. Then it is necessary to analyze two cases:

1. $b<a$. This is the case 1 in Fig. 11 i.e. when the oscillator $B$ has fired before the oscillator $A$. Let us follow the dynamics of the system in real time. The nearest event, that affects on the further behavior of the entire system, is an appearance of the pulse $A$ at time $a$. Let us stop on at this moment and make the forecast. To this end we define a concept of the moments of expected firings of the oscillators, i.e. $a^{e}$ and $b^{e}$. Let us imagine that we have shifted back in time with respect to the moment a. Since the oscillator $A$ has not fired yet, one should expect the appearance of the next $A$ and $B$ pulses at the moments $a^{e}=a$ and $b^{e}=b+T_{b}$ respectively, where $T_{b}$ is the period of $B$. We call this situation as " $A$ fires and $B$ is at an expected state" and denote symbolically as $\left(a, b^{e}\right)$. Now we consider the instant $a$. Since $A$ fires, the next expected values can be transformed to

$$
\begin{array}{ll}
a_{\text {next }}^{e}=a+T_{a} & =a^{e}+T_{a}, \\
b_{\text {next }}^{e}=b+T_{b}+\Delta_{b}\left(\varphi_{b}, \varepsilon_{b}\right) & =b^{e}+\Delta_{b}\left(\varphi_{b}, \varepsilon_{b}\right),
\end{array}
$$

where $\Delta_{b}\left(\varphi_{b}, \varepsilon_{b}\right)$ is the time shift of the oscillator $B$ due to the impact of $A$. It depends on the phase $\varphi_{b}$ of the pacemaker $A$ with respect to $B$ and the influence strength $\varepsilon_{b}$. The phase $\varphi_{b}$ can be calculated as follows

$$
\varphi_{b}=\frac{a-b}{T_{b}}(\bmod 1)
$$


or, in terms of the expected values,

$$
\varphi_{b}=\frac{a^{e}-b^{e}}{T_{b}}(\bmod 1) .
$$

Note that the phase $\varphi_{b}$ is a positive value, and it belongs to the $[0,1]$ segment (negative values in the two previous expressions are eliminated by the mod 1 operation). To determine which oscillator fires next, one should compare $a_{\text {next }}^{e}$ and $b_{\text {next }}^{e}$. If $a_{\text {next }}^{e}<b_{\text {next }}^{e}$, then $A$ fires and $B$ remains at an expected state until $b_{\text {next }}^{e}$, i.e. the system moves to the state $\left(a, b^{e}\right)_{n e x t}$. Otherwise, if $b_{n e x t}^{e}<a_{n e x t}^{e}$, then $B$ fires, and $A$ jumps to an expected state and the entire system's state becomes $\left(a^{e}, b\right)_{n e x t}$.

2. $b>a$. This is the case 2 in Fig. 1. This inverse situation is analogous to the previous one with the difference in speculations owing to the firing of the pacemaker $A$ prior to $B$. Then expected values $a^{e}$ and $b^{e}$ can be written accordingly: $a^{e}=a+T_{a}$ and $b^{e}=b$. One can call this case as " $B$ fires and $A$ is at an expected state" and denote by $\left(a^{e}, b\right)$. The next expected values are given by the following expression:

$$
\begin{aligned}
& a_{\text {next }}^{e}=a^{e}+\Delta_{a}\left(\varphi_{a}, \varepsilon_{a}\right) \\
& b_{\text {next }}^{e}=b^{e}+T_{b}
\end{aligned}
$$

where $\Delta_{a}\left(\varphi_{a}, \varepsilon_{a}\right)$ is the time shift of the oscillator $A$. It depends on the phase of the oscillator $B$ with respect to $A$, i.e. $\varphi_{a}=\left(b^{e}-a^{e}\right) / T_{a}(\bmod 1)$, and the influence strength $\varepsilon_{a}$. Further analysis is also similar to the case 1 . Namely, if $b_{\text {next }}^{e}<a_{\text {next }}^{e}$, then $B$ fires and $A$ jumps to the expected state $a_{n e x t}^{e}$, i.e. the system moves to the state $\left(a^{e}, b\right)_{n e x t}$. Otherwise, if $a_{\text {next }}^{e}<b_{\text {next }}^{e}$, then $A$ fires and $B$ remains in the expected state and the system state becomes $\left(a, b^{e}\right)_{n e x t}$.

Summarizing the above calculations, the model can be represented by the following scheme:

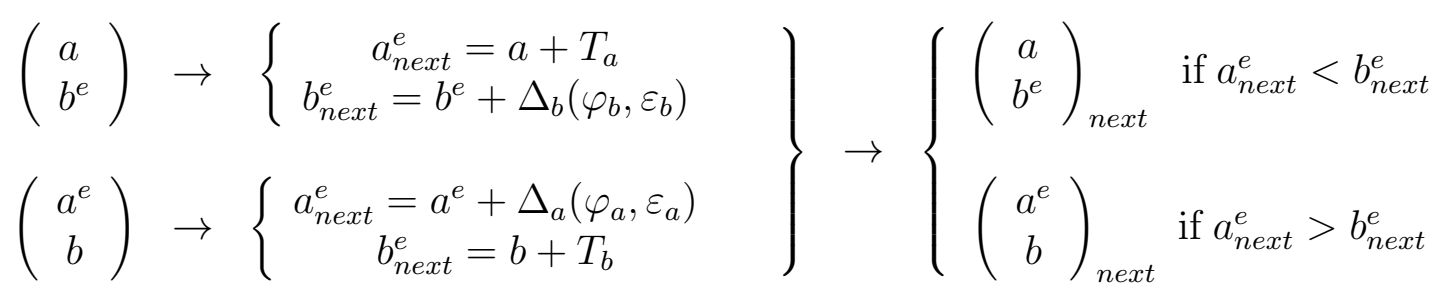

In the notions of the expected values, which we denote for convenience as $a^{e} \equiv \hat{a} b^{e} \equiv \hat{b}$, 
the dynamics can be described by the following difference equation:

$$
\left(\begin{array}{l}
\widehat{a}_{n+1} \\
\widehat{b}_{n+1}
\end{array}\right)=\left(\begin{array}{c}
\widehat{a}_{n} \\
\widehat{b}_{n}
\end{array}\right)+\left\{\begin{array}{l}
\left(\begin{array}{c}
T_{a} \\
\Delta_{b}\left(\varphi_{n}^{b}, \varepsilon_{b}\right)
\end{array}\right), \widehat{a}_{n}<\widehat{b}_{n}, \text { and then } A \text { fires at time } \widehat{a}_{n} \\
\left(\begin{array}{l}
\Delta_{a}\left(\varphi_{n}^{a}, \varepsilon_{a}\right) \\
T_{b}
\end{array}\right), \widehat{b}_{n}<\widehat{a}_{n}, \text { and then } B \text { fires at time } \widehat{b}_{n}
\end{array}\right.
$$

where:

$$
\varphi_{n}^{a}=\frac{\left(\widehat{b}_{n}-\widehat{a}_{n}\right)}{T_{a}}(\bmod 1), \quad \varphi_{n}^{b}=\frac{\left(\widehat{a}_{n}-\widehat{b}_{n}\right)}{T_{b}}(\bmod 1) .
$$

To simulate the dynamics of two interacting oscillators coupled by PRCs, it is necessary to carry out the iterative process (3) for the expected pulses and put sequentially in the time scale the firing moments of the oscillators $A$ and $B$ depending on the result of the comparison $\widehat{a}_{n}$ and $\widehat{b}_{n}$. An investigation of the case of two interacting pacemakers in more detail with the description of the possible modes of behavior is given in Section 3.1.

\subsection{Derivation of the basic model equation}

Assume that there are $N$ autonomous pulse oscillators (or pacemakers). Suppose that all the pacemakers are different. This means that each has its own intrinsic cycle length $T_{i}, i=1, \ldots, N$, and the beating amplitude. To define coupling between pacemakers, it is necessary to determine the topology of the system space. In other words, one should specify the nearest neighbors of each pacemaker in the space. Vice versa, it is obvious that the determination of coupling between the elements of such spatially discrete system sets its topology.

First of all we develop the general model of $N$ mutually coupled pulse oscillators. Suppose that all the pacemakers interact with each other, i.e. so-called global coupling is realized. The model derived in Section 2.1 can be easily generalized to the case of $N$ pacemakers. We operate with the expected values introduced in Section 2.1, the real firings of the pacemakers are found by the analysis of the expected impacts series.

Let the set of expected firings $\left\{\widehat{a}_{i}\right\}_{1, \ldots, N}$ be located in a time axis (see Fig. 2). This means that in the absence of coupling, pacemakers strike at these instants. Suppose now that some oscillator acts on another one by means of the $\operatorname{PRC}\left\{\Delta_{i j}\left(\varphi^{i j}, \varepsilon_{i j}\right)\right\}_{1, \ldots, N}$, where $\varphi^{i j}$ is the phase of the $j$-th pacemaker with respect to the $i$-th one and $\varepsilon_{i j}$ is a total parameter defining coupling between the $j$-th and $i$-th elements. 
The next values of the expected firings can be calculated by the same manner as in Section 2.1. Because the $j$-th oscillator appears before all others (see. Fig. 2), it does not undergo any influence and fires as a real impact of the $j$-th pacemaker. Thus, the given event makes the shifts of all other oscillators according to the set of the PRCs $\left\{\Delta_{i j}\right\}$. The $j$-th oscillator is shifted to a new expected moment as an unperturbed one, i.e. by adding its own cycle length $T_{j}$. To get the next sequential expected values, one should make the same procedure with the newly obtained expected pulses. The dynamics of the system can be easily represented as the following iterative relation:

$$
\widehat{a}_{n+1}^{i}=\widehat{a}_{n}^{i}+\left\{\begin{array}{lc}
T_{i}, & i=j, \\
\Delta_{i j}\left(\varphi_{n}^{i j}, \varepsilon_{i j}\right), & i \neq j,
\end{array} \quad j: a_{n}^{j}=\min \left\{a_{n}^{i}\right\}_{i=1, \ldots, N},\right.
$$

where

$$
\varphi_{n}^{i j}=\frac{\widehat{a}_{n}^{j}-\widehat{a}_{n}^{i}}{T_{i}}(\bmod 1),
$$

It is convenient to rewrite the PRCs by normalizing $\left\{\Delta_{i j}\right\}$ on the intrinsic pacemaker cycle lengths $T_{i}$ and to define as follows:

$$
\Delta_{i j}\left(\varphi_{n}^{i j}, \varepsilon_{i j}\right)=\left\{f_{i j}\left(\varphi_{n}^{i j}, \varepsilon_{i j}\right) \cdot T_{i}\right\}, \varphi_{n}^{i j} \in[0,1]
$$

where $\left\{f_{i j}\left(\varphi_{n}^{i j}, \varepsilon_{i j}\right)\right\}$ are the dimensionless functions, which are also called the PRCs. The real pacemakers can have identical nature but differ in the intrinsic cycle lengths. Then the form of dimensionless PRCs is identical for them, i.e. their $f(\varphi, \varepsilon)$ coincide, while $\Delta(\varphi, \varepsilon)$ are different. Moreover, it is convenient to use the functions $\left\{f_{i j}\left(\varphi_{n}^{i j}, \varepsilon_{i j}\right)\right\}$ in the construction of the equations for the dimensionless phase differences between pacemaker pairs. In Section 3 we will demonstrate this approach for the systems of two and three coupled pulse oscillators.

\section{Applications}

\subsection{Two interacting pulse oscillators}

Let us investigate the system of two interacting pacemakers in detail. As well as in Section 2.1, suppose that the system consists of two oscillators $A$ and $B$ coupled by means of the phase response curves $\Delta_{a}\left(\varphi_{n}^{a}, \varepsilon_{a}\right)$ and $\Delta_{b}\left(\varphi_{n}^{b}, \varepsilon_{b}\right)$. It is clear that the map (3) specifying the dynamics of such a system is a particular case of the general model (4) restricted to 
$N=2$. Rewrite (3) using the expressions for the dimensionless PRCs:

$$
\left(\begin{array}{l}
\widehat{a}_{n+1} \\
\widehat{b}_{n+1}
\end{array}\right)=\left(\begin{array}{c}
\widehat{a}_{n} \\
\widehat{b}_{n}
\end{array}\right)+\left\{\begin{array}{l}
\left(\begin{array}{l}
T_{a} \\
f_{b}\left(\varphi_{n}^{b}, \varepsilon_{b}\right) T_{b}
\end{array}\right), \widehat{a}_{n}<\widehat{b}_{n}, \text { and then } A \text { fires at time } \widehat{a}_{n}, \\
\left(\begin{array}{l}
f_{a}\left(\varphi_{n}^{a}, \varepsilon_{a}\right) T_{a} \\
T_{b}
\end{array}\right), \widehat{b}_{n}<\widehat{a}_{n}, \text { and then } B \text { fires at time } \widehat{b}_{n}
\end{array}\right.
$$

where:

$$
\varphi_{n}^{a}=\frac{\left(\widehat{b}_{n}-\widehat{a}_{n}\right)}{T_{a}}(\bmod 1), \varphi_{n}^{b}=\frac{\left(\widehat{a}_{n}-\widehat{b}_{n}\right)}{T_{b}}(\bmod 1) .
$$

Let us assume that the pacemakers have one the same nature. Hence, one can accept $f_{a}(\varphi, \varepsilon) \equiv f_{b}(\varphi, \varepsilon) \equiv f(\varphi, \varepsilon)$, where $f(\varphi, \varepsilon)$ is an one-parametric function. The parameter $\varepsilon$ integrally determines a total influence of one oscillator on another one. In the symmetric case we have $\varepsilon_{a}=\varepsilon_{b} \equiv \varepsilon$. In our further consideration we do not accept this symmetry.

From the mathematical point of view the system (5) is a map of the real plane into itself depending on four parameters: $T_{a}, T_{b}, \varepsilon_{a}$ and $\varepsilon_{b}$. It is easy to comprehend that the effective parameter that changes the behavior of the system (5) is the dimensionless ratio $\delta=T_{b} / T_{a}$. Specifying parameters $\varepsilon_{a}, \varepsilon_{b}, \delta$ and the function $f(\varphi, \varepsilon)$, one can iterate directly the equations (5) marking on the time axis the values of the real firings of the pacemakers $A$ and $B$. Some examples of this simulation are presented below.

In spite of the fact that the expression (5) defines the two-dimensional map, the values $\widehat{a}_{n}$ and $\widehat{b}_{n}$ increase gradually because they represent the time series of the expected firings of the oscillators. Therefore, trajectories of the map (5) are infinite. Thus, this is not informative for us. It is more important to derive a map that determines the dynamics of the phase difference of the pacemakers.

Let us introduce the dimensionless phase difference of the pacemakers $A$ and $B$ :

$$
x_{n}=\frac{\widehat{a}_{n}-\widehat{b}_{n}}{T_{a}} .
$$

The choice of $T_{a}$ as a normalization factor is not essential. Having selected $T_{b}$, as a result we obtain the similar expressions. Subtracting the second equation of the system (5) from the first one and dividing the result by $T_{a}$ we get the following:

$$
x_{n+1}= \begin{cases}x_{n}+1-\delta f\left(\frac{-x_{n}}{\delta}(\bmod 1), \varepsilon_{b}\right), & x_{n}<0 \\ x_{n}+f\left(x_{n}(\bmod 1), \varepsilon_{a}\right)-\delta, & x_{n}>0\end{cases}
$$


where $\delta=T_{b} / T_{a}$. Here we take into account that $x_{n}<0$ if $\widehat{a}_{n}<\widehat{b}_{n}$ and $x_{n}>0$ if $\widehat{a}_{n}>\widehat{b}_{n}$.

Let us make a brief analysis of the developed map. Because in general $x \in(-\infty ; \infty)$, the equation (6) represents a one-dimensional nonlinear map of the real axis into itself. Note that the map can not be reduced to a circle map by the restriction of $x$ to the range $[0 ; 1]$ as it is usually done for two pacemakers interacting by PRCs. It is essentially asymmetric with respect to changing $x$ to $-x$ (see Fig. [3). If $f(x, \varepsilon)$ is a nonmonotonic function, then the map (6) is nonlinear, and it can exhibit a big variety of the behavior: from complex periodic motion to chaotic dynamics. Owing to $x \in(-\infty ; \infty)$, in a rigorous sense it is not a difference of phases of the pacemakers. In this context one can call $x$ as the generalized phase difference. Analyzing Eq. (6) one can find out which oscillator, $A$ or $B$, fires at the given discrete time $n$. This depends on the sign of $x$ : $A$ fires if $x_{n}<0$ and $B$ fires when $x_{n}>0$. Thus, it makes possible to determine the phase-locking degree of the pacemakers. However, taking into consideration only the values $x_{n}$, we may not reconstruct the initial time series of firing events of the pacemakers $A$ and $B$. Using $x_{n}$, one can say only about their phase difference.

Let us show how the model equations (5), (6) can be applied to the investigation of the behavior of two interacting pacemakers.

Analysis of real systems [Glass et al., 1987] shows that the function $f(x, \varepsilon)$ can take different forms. But, as a rule, it obeys a number of general properties. For example, $f(0, \varepsilon)=f(1, \varepsilon)=0$. Usually it has a maximum and a minimum. Sometimes instead of extrema it has breaks. Let the function $f(x, \varepsilon)$ be in an elementary form that is often used (see, e.g. [Glass \& Zeng, 1990]). Namely, let $f(x, \varepsilon)=\varepsilon \sin 2 \pi x$. This leads to an array of dynamical equations:

$$
\begin{gathered}
\left(\begin{array}{l}
\widehat{a}_{n+1} \\
\widehat{b}_{n+1}
\end{array}\right)=\left(\begin{array}{l}
\widehat{a}_{n} \\
\widehat{b}_{n}
\end{array}\right)+\left\{\begin{array}{l}
\left(\begin{array}{l}
T_{a} \\
\varepsilon_{b} \sin \left(2 \pi \varphi_{n}^{b}\right) T_{b}
\end{array}\right), \widehat{a}_{n}<\widehat{b}_{n} \\
\left(\begin{array}{l}
\varepsilon_{a} \sin \left(2 \pi \varphi_{n}^{a}\right) T_{a} \\
T_{b}
\end{array}\right), \widehat{b}_{n}<\widehat{a}_{n} .
\end{array}\right. \\
x_{n+1}= \begin{cases}x_{n}+1-\delta \varepsilon_{b} \sin \left(\frac{-x_{n}}{\delta}(\bmod 1) \cdot 2 \pi\right), & x_{n}<0 \\
x_{n}+\varepsilon_{a} \sin \left(x_{n}(\bmod 1) \cdot 2 \pi\right)-\delta, & x_{n}>0\end{cases}
\end{gathered}
$$

In Fig. 3 examples of direct simulation of the system (77), (8) are presented. In the left column some possible phase-lockings found on the basis of Eq. (7) are displayed. In 
the right column the corresponding map (8), its periodic orbit and values of Lyapunov exponents are shown. Fig. $3 \mathrm{~d}$ represents the existence of the chaotic behavior for the system at $\varepsilon_{a}=0.1205, \varepsilon_{b}=1.2845$ and $\delta=0.6465$.

As a conclusion of this section it should be noted that the system of two bidirectionally acting pacemakers has been already intensively investigated (see, e.g., [Ikeda et al., 2004] and references herein) as a qualitative model of cardiac arrhythmia known as modulated parasystole. The interacting oscillators represented the pairs of cardiac pacemakers such as: the sinoatrial (SA) node and the ventricle contracting by different factors, e.g. ectopic pacemaker, etc. Hereby the authors used various kinds of PRCs $f(x, \varepsilon)$ approximating the experimental data on stimulating the cardiac cells of animals by single electric current pulses. Recently in the paper [Loskutov et al., 2004] the system of two interacting pacemakers similar to (17), (8) has been analyzed. In this paper taking into account the refractory time the various types of the smooth PRCs were examined and the bifurcation diagrams of possible phase-lockings were constructed. However, in [Loskutov et al., 2004] the behavior regimes when the firings of the pacemakers are not alternated, i.e. cases of strong discrepancies in the intrinsic cycle length $\left(T_{a} \gg T_{b}\right.$ and vice versa), were not investigated. The system (17), (8) is more general and takes into account all possible variants.

\subsection{Three pacemakers}

Below we explain why the special case of three interacting oscillators is worth individual attention. First, as is known there are three drivers of the rhythm in the cardiac conductive system: the SA node is a leading pacemaker, the AV junction and Purkinje fibers are latent pacemakers, which under normal conditions are suppressed by the sinus rhythm. However, at violations of conductivity and pulse initiation, the cardiac pacemakers can influence on each other, i.e. so-called bidirectional coupling may be realized. Second, in pathology a group of contractile cardiomyocytes can also initiate action potentials: an ectopic (abnormal) cardiac pacemaker may emerge and start to compete with the SA node for leading the heart rhythm. Third, at stimulating the cardiac tissue by external current impulses (cardio-stimulation), the cardiac rate is changed. The external stimulaters can be naturally included in our general model of interacting pacemakers as additional leading pulse oscillators. 
Thus, representation of the heart conductive system as at least three coupled autonomous oscillators (Fig. (4) is very useful for understanding which influence of an additional pacemaker exerts on a system of two bidirectionally interacting drivers of the rhythm (i.e. the SA and AV nodes) considered above. Investigation of the possible behavior modes of a larger amount of interacting pacemakers turns out to be a very complicated both analytical (mathematical) and numerical problem. For example for a system of five coupled pulse oscillators we have 25 various functions and 29 different parameters (25 values of $\varepsilon_{i j}$ and 4 independent ratios $T_{i} / T_{j}$ ). This is due to the fact that, in general, the cardiac pacemakers have various frequencies (or intrinsic cycle lengths $T_{i}$ ) and the different nature. This means that the PRCs $\left\{f_{i j}\left(x, \varepsilon_{i j}\right)\right\}$ determining coupling between a pair of pacemakers have different forms. For some heart pacemakers PRCs have been measured by the direct experiments on cardiomyocytes of animals (see [Glass et al., 1986]). Other PRCs can be chosen using general principles based on the nature of nodes or on the basis of the collateral measurements [Ikeda et al., 1988].

Applying the general model equations (44) for three interacting pacemakers, one can get the following system:

$$
\left(\begin{array}{c}
\widehat{a}_{n+1} \\
\widehat{b}_{n+1} \\
\widehat{c}_{n+1}
\end{array}\right)=\left(\begin{array}{c}
\widehat{a}_{n} \\
\widehat{b}_{n} \\
\widehat{c}_{n}
\end{array}\right)+\left\{\begin{array}{c}
\left(\begin{array}{c}
T_{a} \\
\Delta_{b a}\left(\varphi_{n}^{b a}, \varepsilon_{b a}\right) \\
\Delta_{c a}\left(\varphi_{n}^{c a}, \varepsilon_{c a}\right)
\end{array}\right), \text { if } \widehat{a}_{n}<\widehat{b}_{n}, \widehat{c}_{n} ; \quad A \text { fires at time } \widehat{a}_{n} \\
\left(\begin{array}{c}
\Delta_{a b}\left(\varphi_{n}^{a b}, \varepsilon_{a b}\right) \\
T_{b}\left(\varphi_{n}^{c b}, \varepsilon_{c b}\right) \\
\Delta_{c b}(\varphi) \\
\Delta_{a c}\left(\varphi_{n}^{a c}, \varepsilon_{a c}\right) \\
\Delta_{b c}\left(\varphi_{n}^{b c}, \varepsilon_{b c}\right) \\
T_{c}
\end{array}\right), \text { if } \widehat{c}_{n}<\widehat{a}_{n}, \widehat{b}_{n} ; \quad C \text { fires at time } \widehat{c}_{n}, \widehat{c}_{n} ; \quad B \text { fires at time } \widehat{b}_{n}
\end{array}\right.
$$

where

$$
\begin{aligned}
\varphi_{n}^{b a} & =\frac{\widehat{a}_{n}-\widehat{b}_{n}}{T_{b}}(\bmod 1), & \varphi_{n}^{c a} & =\frac{\widehat{a}_{n}-\widehat{c}_{n}}{T_{c}}(\bmod 1) \\
\varphi_{n}^{a b} & =\frac{\widehat{b}_{n}-\widehat{a}_{n}}{T_{a}}(\bmod 1), & \varphi_{n}^{c b} & =\frac{\widehat{b}_{n}-\widehat{c}_{n}}{T_{c}}(\bmod 1) \\
\varphi_{n}^{a c} & =\frac{\widehat{c}_{n}-\widehat{a}_{n}}{T_{a}}(\bmod 1), & \varphi_{n}^{b c} & =\frac{\widehat{c}_{n}-\widehat{b}_{n}}{T_{b}}(\bmod 1)
\end{aligned}
$$

The response functions $\Delta_{i j}$ are supposed to have a form $\Delta_{i j}\left(\varphi_{n}^{i j}, \varepsilon_{i j}\right)=f_{i j}\left(\varphi_{n}^{i j}, \varepsilon_{i j}\right) T_{i}, \varphi_{n}^{i j} \in$ $[0 ; 1], i, j=a, b, c$. The example of a system of three bidirectionally interacting cardiac 
pacemakers: the SA node, the AV junction and ectopic pacemaker, is shown in Fig. 四.

It is convenient to study Eqs. (9) introducing phase differences of the pacemakers. Let us define,

$$
x_{n}=\frac{\widehat{a}_{n}-\widehat{b}_{n}}{T_{b}}, \quad y_{n}=\frac{\widehat{c}_{n}-\widehat{b}_{n}}{T_{b}}, \quad \alpha=\frac{T_{a}}{T_{b}}, \quad \beta=\frac{T_{c}}{T_{b}} .
$$

Then for the phase differences we obtain the map of the real plane into itself:

$$
\begin{aligned}
& \left(\begin{array}{l}
x_{n+1} \\
y_{n+1}
\end{array}\right)=\left(\begin{array}{c}
x_{n} \\
y_{n}
\end{array}\right)+ \\
& +\left\{\begin{array}{l}
\left(\begin{array}{l}
\alpha-f_{b a}\left(\left\{x_{n}\right\}, \varepsilon_{b a}\right) \\
\beta f_{c a}\left(\left\{\frac{x_{n}-y_{n}}{\beta}\right\}, \varepsilon_{c a}\right)-f_{b a}\left(\left\{x_{n}\right\}, \varepsilon_{b a}\right)
\end{array}\right), \quad x_{n}<0, x_{n}<y_{n} \\
\left(\begin{array}{l}
\alpha f_{a b}\left(\left\{\frac{-x_{n}}{\alpha}\right\}, \varepsilon_{a b}\right)-1 \\
\beta f_{c b}\left(\left\{\frac{-y_{n}}{\beta}\right\}, \varepsilon_{c b}\right)-1
\end{array}\right), \quad x_{n}>0, y_{n}>0 \\
\left(\begin{array}{l}
\alpha f_{a c}\left(\left\{\frac{y_{n}-x_{n}}{\alpha}\right\}, \varepsilon_{a c}\right)-f_{b c}\left(\left\{y_{n}\right\}, \varepsilon_{b c}\right) \\
\beta-f_{b c}\left(\left\{y_{n}\right\}, \varepsilon_{b c}\right)
\end{array}\right), \quad y_{n}<x_{n}, y_{n}<0
\end{array}\right.
\end{aligned}
$$

Here we denote $\{x\}$ as the fractional part of $x$. The conditions in the right hand side of (10) divide the plane into three areas corresponding to the firing pacemaker (see Fig. 國). The map (10) has breaks on the boundaries of these areas.

Suppose that the pacemaker $B$ is an external stimulus (see Fig. [b). Then the expressions (92), (10) become simpler. The stimulus $B$ acts on the pacemakers $A$ and $C$ but does not experience any influence. In this situation $f_{b a}\left(x, \varepsilon_{b a}\right) \equiv f_{b c}\left(x, \varepsilon_{b c}\right) \equiv 0$ and the model (9), (10) can be rewritten as follows:

$$
\left(\begin{array}{l}
\widehat{a}_{n+1} \\
\widehat{b}_{n+1} \\
\widehat{c}_{n+1}
\end{array}\right)=\left(\begin{array}{l}
\widehat{a}_{n} \\
\widehat{b}_{n} \\
\widehat{c}_{n}
\end{array}\right)+\left\{\begin{array}{l}
\left(\begin{array}{l}
T_{a} \\
0 \\
\Delta_{c a}\left(\varphi_{n}^{c a}, \varepsilon_{c a}\right)
\end{array}\right), \text { if } \widehat{a}_{n}<\widehat{b}_{n}, \widehat{c}_{n} ; \quad A \text { fires at time } \widehat{a}_{n} \\
\left(\begin{array}{l}
\Delta_{a b}\left(\varphi_{n}^{a b}, \varepsilon_{a b}\right) \\
T_{b} \\
\Delta_{c b}\left(\varphi_{n}^{c b}, \varepsilon_{c b}\right)
\end{array}\right), \text { if } \widehat{b}_{n}<\widehat{a}_{n}, \widehat{c}_{n} ; \quad B \text { fires at time } \widehat{b}_{n} \\
\left(\begin{array}{l}
\Delta_{a c}\left(\varphi_{n}^{a c}, \varepsilon_{a c}\right) \\
0 \\
T_{c}
\end{array}\right), \text { if } \widehat{c}_{n}<\widehat{a}_{n}, \widehat{b}_{n} ; \quad C \text { fires at time } \widehat{c}_{n}
\end{array}\right.
$$


The corresponding expression for the phase differences takes the form:

$$
\begin{aligned}
& \left(\begin{array}{l}
x_{n+1} \\
y_{n+1}
\end{array}\right)=\left(\begin{array}{l}
x_{n} \\
y_{n}
\end{array}\right)+
\end{aligned}
$$

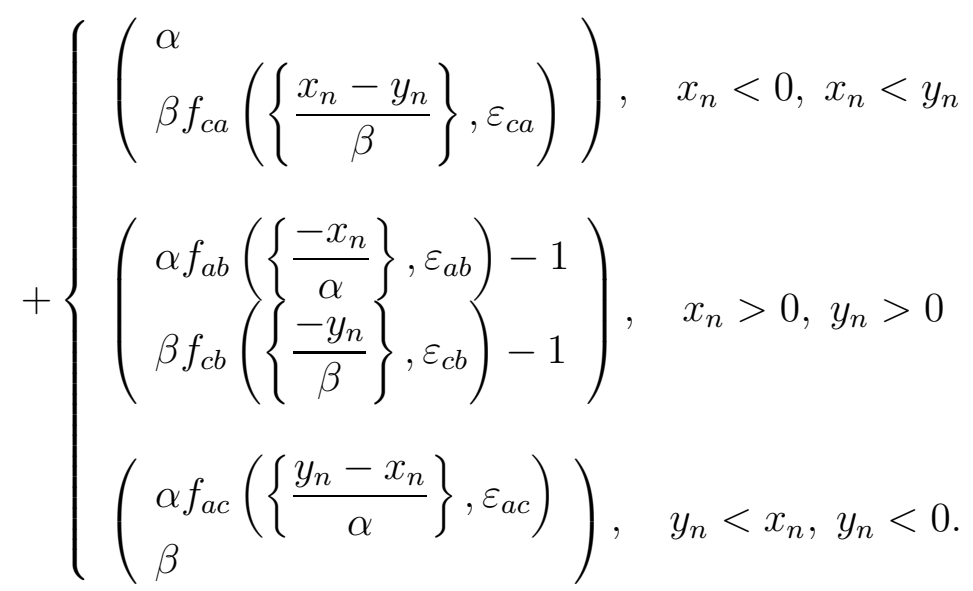

In Fig. 5]some examples of phase-lockings and complex dynamics for the system (91), (10) are shown. The PRCs are chosen in the sinus form, i.e. $f_{i j}\left(x, \varepsilon_{i j}\right)=\varepsilon_{i j} \sin (2 \pi x) . T_{i}$ and $\varepsilon_{i j}$ are indicated in the legends. It is obvious that the model of three interacting pacemakers requires further investigation in detail. In particular, we believe that appropriate choice of influence strength and period of external stimulation in the equations (12) will remove the system from undesirable complex behavior to the normal heart rhythm. It will be represented within forthcoming works.

\section{Lattices of Coupled Pulse Oscillators}

Let us demonstrate briefly the way how one can approximate discrete distributed media on the basis of the general model of coupled oscillators (44). Considering a cardiac pacemaker on the microscopic level, one can represent it as a large group of cells, which generate the heart rhythm and synchronize their action potentials to initiate the cardiac contraction. Thus, instead of examining a single pacemaker we should construct a lattice of coupled pulse oscillators. In this paper we restrict ourselves to the one- (chain) and two-dimensional (lattice) cases.

Let us suppose that the autonomous pacemakers are located in sites of the two-dimensional square lattice of the size $N \times M$. An element of the lattice with coordinates $(i, j)$ is designated as $A^{i, j}$, where $i=1, \ldots, N$ and $j=1, \ldots, M$. We restrict consideration to the 
homogeneous medium and accept a number of limitations on anisotropy. This means that the pacemakers of the lattice are identical, i.e. they have the same cycle length $T_{i, j} \equiv T$, $i=1, \ldots, N, j=1, \ldots, M$ (however, in fact, cells from the periphery of the sinus pacemaker show the shortest cycle length, although the centre acts as the leading pacemaker site). This limitation decreases a quantity of parameters of the system and hence simplifies the study of the model. Now we should define coupling between elements. In works devoted to the coupled map lattices (CML) two main types of coupling are usually assumed: the nearest neighbors and global couplings (see, e.g., [Kaneko, 1989]). Since in the previous sections we supposed that pacemakers interact with each other, this time as an example we consider lattices with the coupling to nearest neighbors: first, a two-dimensional lattice, and then a chain of coupled pulse oscillators.

Within the square lattice each pacemaker $A^{i, j}$ interacts with four of the neighbors according to the schematic picture in Fig. 6a. Taking into account the limitation of homogeneous medium, one can assert that all couplings of the lattice are identical, i.e. each two adjacent elements interact with each other by a general law defined by the identical PRCs $f(x, \varepsilon)$. Moreover, suppose that the coupling between a pair of elements is isotropic in such a sense that $\varepsilon_{(i, j)\left(i^{\prime}, j^{\prime}\right)}=\varepsilon_{\left(i^{\prime}, j^{\prime}\right)(i, j)}$, and is equal to one of the values $\varepsilon_{1}$ or $\varepsilon_{2}$ depending on the relative position of elements. This means that there is an anisotropy of the influence strength in vertical and horizontal directions. In other words, if two pacemakers are the neighbors in the vertical direction, they interact by $f\left(x, \varepsilon_{1}\right)$, and if they are horizontal neighbors, then they are coupled by $f\left(x, \varepsilon_{2}\right)$.

Note that all these restrictions are made for the simplification of the analytical form of the resulting model. In general, it is possible to write the expressions for a two-dimensional lattice of coupled pulse oscillators without any limitations. It is a subject of a standalone investigation and lies beyond the framework of the given study.

Let us write equations that determine the iterative dynamics of the expected firings of the pacemakers $\left\{\widehat{a}^{i, j}\right\}_{\substack{i=1, \ldots, N \\ j=1, \ldots, M}}$ on the basis of the approach represented in Sec.2.2. To obtain the $(n+1)$-th value of the individual element $\widehat{a}^{i, j}$, it is necessary to analyze all elements of the lattice since they are coupled with each other by means of a local coupling. In other words, the considered element cannot be affected by others at the $n$-th time step because the latter is suppressed by the influence of other elements and remains at the expected state 
until the $(n+1)$-th step. Then dynamics of the model can be described by the following expression:

$$
\widehat{a}_{n+1}^{i, j}=\widehat{a}_{n}^{i, j}+ \begin{cases}T, & \text { if } \widehat{a}_{n}^{i, j}=\widehat{a}^{\min }, \\ f\left(\varphi_{n}^{(i, j)(0,+1)}, \varepsilon_{1}\right) \cdot T, & \text { if } \widehat{a}_{n}^{i, j+1}=\widehat{a}^{\min }, \\ f\left(\varphi_{n}^{(i, j)(0,-1)}, \varepsilon_{1}\right) \cdot T, & \text { if } \widehat{a}_{n}^{i, j-1}=\widehat{a}^{\min }, \\ f\left(\varphi_{n}^{(i, j)(+1,0)}, \varepsilon_{2}\right) \cdot T, & \text { if } \widehat{a}_{n}^{i+1, j}=\widehat{a}^{\min }, \\ f\left(\varphi_{n}^{(i, j)(-1,0)}, \varepsilon_{2}\right) \cdot T, & \text { if } \widehat{a}_{n}^{i-1, j}=\widehat{a}^{\min }, \\ 0, & \text { otherwise }\end{cases}
$$

where the phases are

$$
\begin{aligned}
& \varphi_{n}^{(i, j)(0,+1)}=\left\{\frac{\widehat{a}_{n}^{i, j+1}-\widehat{a}_{n}^{i, j}}{T}\right\} \\
& \varphi_{n}^{(i, j)(0,-1)}=\left\{\frac{\widehat{a}_{n}^{i, j-1}-\widehat{a}_{n}^{i, j}}{T}\right\} \\
& \varphi_{n}^{(i, j)(+1,0)}=\left\{\frac{\widehat{a}_{n}^{i+1, j}-\widehat{a}_{n}^{i, j}}{T}\right\} \\
& \varphi_{n}^{(i, j)(-1,0)}=\left\{\frac{\widehat{a}_{n}^{i-1, j}-\widehat{a}_{n}^{i, j}}{T}\right\}
\end{aligned}
$$

The constructed model demands detailed investigation on the basis of the approach developed for the CML (see, e.g., [Kaneko, 1989]). It will be represented in the succeeding works.

As the second example let us consider a chain of the identical pulse oscillators coupled by the nearest neighbor principle. We restrict ourselves to a homogeneous case with anisotropy of the right and left direction in the influence strength between the nearest neighbors. The schematic picture of the chain is shown in Fig. 6b. Similarly to the above consideration one can get:

$$
\widehat{a}_{n+1}^{i}=\widehat{a}_{n}^{i}+\left\{\begin{array}{ll}
T, & \text { if } \widehat{a}_{n}^{i}=\widehat{a}^{\mathrm{min}} \\
f\left(\varphi_{n}^{i,+1}, \varepsilon_{2}\right) \cdot T, & \text { if } \widehat{a}_{n}^{i+1}=\widehat{a}^{\mathrm{min}}, \\
f\left(\varphi_{n}^{i,-1}, \varepsilon_{1}\right) \cdot T, & \text { if } \widehat{a}_{n}^{i-1}=\widehat{a}^{\mathrm{min}}, \\
0, & \text { otherwise }
\end{array} \widehat{a}^{\min }=\min \left\{\widehat{a}_{n}^{i}\right\}_{i=1 \ldots N}\right.
$$

where the phases are the following

$$
\varphi_{n}^{i,+1}=\left\{\frac{\widehat{a}_{n}^{i+1}-\widehat{a}_{n}^{i}}{T}\right\} \quad \varphi_{n}^{i,-1}=\left\{\frac{\widehat{a}_{n}^{i-1}-\widehat{a}_{n}^{i}}{T}\right\} .
$$

If $\varepsilon_{1}=0$ (or $\varepsilon_{2}=0$ ), then Eqs (14) define the so-called open-flow model [Willeboordse \& Kaneko, 1994]. 
Because in this work we present a general approach of developing models without the detailed analysis of their behavior, the type of boundary conditions for both lattices has not been indicated. Hence, to investigate such systems analytically or numerically, one should set the boundary conditions along with the PRCs $f(x, \varepsilon)$. Usually the boundary conditions are chosen as periodic, i.e. $\widehat{a}^{i, j+M} \equiv \widehat{a}^{i, j} ; \widehat{a}^{i+N, j} \equiv \widehat{a}^{i, j}$ for the two-dimensional lattice and $\widehat{a}^{i+N} \equiv \widehat{a}^{i}$ for the one-dimensional one. For the open-flow model a condition of the fixed left boundary, $\widehat{a}_{n}^{1} \equiv$ const, is frequently accepted.

The described models (41), (13) and (14) admit generalization to a natural inhomogeneous case by placing different intrinsic cycle lengths of the pacemakers, PRCs and influence strengths for various groups of elements. However, consideration of inhomogeneous anisotropic lattices is extremely difficult problem even for numerical analysis. The first attempts of investigating inhomogeneous lattices of coupled maps (ICML) were described in [Vasil'ev et al., 2000; Loskutov et al., 2002; Rybalko \& Loskutov, 2004].

\section{Summary and Limitations}

In the present study we propose a quite general discrete model of active media by introducing a simple phase response curve interaction between leading centers. We have shown that the PRC can be a useful "tool" for representation of the interaction between pacemakers in cardiac tissue both on a large and small scales. This PRC based model together with demonstrating complex (chaotic) behavior, can describe the entrainment and synchronization phenomena of interacting pulse oscillators. It can also aid to understand their response to an external stimulus with variable intensity and duration (see Fig. 4b), as previously observed in experimental studies [Jalife et al., 1976; Jalife et al., 1980].

Starting with consideration of two interacting pulse oscillators and introducing new concepts of expected values, we have extrapolated our PRC based approach to investigate the mutual influence among an arbitrary large ensemble of pacemakers. The specific cases of the proposed model show that it can be very useful for investigating the dynamical interaction of cardiac nodes.

The last part of our study suggests that the derived general model can be easily applied to construct one- and two--dimensional lattices of active elements interacting by the nearest 
neighbors type. Extension of the model to a three-dimensional case is straightforward.

Finally, some limitations of our approach should be mentioned. First, the proposed model is not complete, there is no a time delay in pulse propagation among pacemakers, which can be very important for describing cardiac arrhythmias. Second, we represented cardiac tissue as a discrete one and used iterative approach to investigate its behavior. However, a large amount of realistic examples of active media is treated as continuous. Nevertheless, cardiac tissue is not a continuum, but is built up by discrete cardiomyocytes (or nodes with approximate dimensions $0.15 \mathrm{~mm} \times 0.02 \mathrm{~mm} \times 0.01 \mathrm{~mm}$ ) [Kuramoto, 1984].

Third and most important, to analyze the essential features governing dynamics of network of active elements, we have not included many important properties of the real conductive cells. These include the relaxation after stimulating, the prolonged (non-peak) form of pulses profiles, realistic topological structure, etc. Further investigations are required to incorporate these features to the general combined model.

\section{Acknowledgements}

This paper was partially supported by INTAS fellowship No 03-55-1920, granted to Ekaterina Zhuchkova. Also, we would like to thank Prof.Alexander Loskutov for critical reading of the manuscript. 


\section{References}

Antzelevitch, C., Jalife, J. \& Moe, G. K. [1982] "Electrotonic modulation of pacemaker activity - further biological and mathematical observations on the behavior of modulated parasystole," Circulation 66(6), 1225-1232.

Bub, G. \& Glass, L. [1994] "Bifurcations in a continuous circle map: A theory for chaotic cardiac arrhythmia," Int. J. Bifurcation and Chaos 5(2), 359-371.

Clayton, R. H. [2001] "Computational models of normal and abnormal action potential propagation in cardiac tissue: linking experimental and clinical cardiology," Physiol. Meas. 22, R15-R34.

Clayton, R. H., Zhuchkova, E. A. \& Panfilov, A. V. [2006] "Phase singularities and filaments: Simplifying complexity in computational models of ventricular fibrillation," Prog. Biophys. Mol. Biol. 90, 378-398.

Courtemanche, M., Glass, L., Belair, J., Scagliotti, D. \& Gordon, D. [1989] "A circle map in a human heart," Physica D 49, 299-310.

Glass, L., Goldberger, A. L. \& Belair, J. [1986] "Dynamics of pure parasystole," Am. J. Physiol. 251(4), H841-H847.

Glass, L., Goldberger, A. L., Courtemanche, M. \& Shrier, A. [1987] "Nonlinear dynamics, chaos and complex cardiac arrhythmias," Proc. R. Soc. London Ser. A-Math. Phys. Eng. Sci. 413(1844), 9-26.

Glass, L. \& Zeng, W. Z. [1990] "Complex bifurcations and chaos in simple theoretical models of cardiac oscillations," Ann. N.Y. Acad. Sci. 591, 316-327.

Glass, L., Nagai, Yo., Hall, K., Talajie, M. \& Nattel, S. [2002] "Predicting the entrainment of reentrant cardiac waves using phase resetting curves," Phys. Rev. E 65, 021908-1-02190810.

Goldberger, A. L. [1990] "Nonlinear dynamics, fractals and chaos: Applications to cardiac electrophysiology," Ann. Biomed. Eng. 18(2), 195-198.

Guevara, M. R. \& Shrier, A. [1987] "Phase resetting in a model of cardiac Purkinje fiber," Biophys. J. 52(2), 165-175.

Ikeda, N. [1982] "Model of bidirectional interaction between myocardial pacemakers based on the phase response curve," Biol. Cybern. 43(3), 157-167. 
Ikeda, N., DeLand, E., Miyahara, H., Takeuchi, A., Yamamoto, H. \& and Sato, T. [1988] “A personal computer-based arrhythmia generator based on mathematical models of cardiac arrhythmia," J. Electrocardiol. 21(Suppl).

Ikeda, N., Takeuchi, A., Hamada, A., Goto, H., Mamorita, N. \& Takayanagi, K. [2004] "Model of bidirectional modulated parasystole as a mechanism for cyclic bursts of ventricular premature contractions," Biol. Cybern. 91(1), 37-47.

Jalife, J. \& Moe, G. K. [1976] "Effects of electronic potentials on pacemaker activity of canine Purkinje fibers in relation to parasystole," Circ. Res. 39(6), 801-808.

Jalife, J., Hamilton, A. J., Lamanna, V. R. \& and Moe, G. K. [1980] "Effects of current flow on pacemaker activity of the isolated kitten sinoatrial node," Am. J. Physiol. 238(3), H307-H316.

Kaneko, K. [1989] "Spatiotemporal chaos in one-dimensional and two-dimensional coupled map lattices," Physica D 37(1-3), 60-82.

Kaneko, K. [1990] "Clustering, coding, switching, hierarchical ordering, and control in a network of chaotic element," Physica D 41(2), 137-172.

Kuramoto, Y. [1984] Chemical Oscillations, Waves, and Turbulence (Springer-Verlag, Berlin). Kuramoto, Y. [1995] "Scaling behavior of turbulent oscillators with nonlocal interaction," Prog. Theor. Phys. 94(3), 321-330.

Loskutov, A., Prokhorov, A. K. \& Rybalko, S. D. [2002] "Dynamics of inhomogeneous chains of coupled quadratic maps," Theor. Math. Phys. 132(1), 983-999.

Loskutov, A., Rybalko, S. \& Zhuchkova, E. [2004] "Model of cardiac tissue as a conductive system with interacting pacemakers and refractory time," Int. J. Bifurcation and Chaos 14(7), 2457-2466.

Reiner, V. S. \& Antzelevich, C. [1985] "Phase resetting and annihilation in a mathematical model of sinus node," Am. J. Physiol. 249, H1143-H1153.

Rybalko, S. \& Loskutov, A. [2004] "Dynamics of inhomogeneous one-dimensional coupled map lattices," http://arxiv.org/abs/nlin.CD/0409014.

Sano, T., Sawanobori, T. \& Adaniya, H. [1978] "Mechanism of rhythm determination among pacemaker cells of the mammalian sinus node," Am. J. Physiol. 235, H379-H384.

Shibata, T. \& Kaneko, K. [1998] "Collective chaos," Phys. Rev. Lett. 81(19), 4116-4119.

Vasil'ev, K. A., Loskutov, A., Rybalko, S. D. \& Udin, D. N. [2000] "Model of a spatially 
inhomogeneous one-dimensional active medium," Theor. Math. Phys. 124(3), 1286-1297. Wiener, N. \& Rosenblueth, A. [1946] "Conduction of impulses in cardiac muscle," Arch. Inst. Cardiol. Mex. 16, 205-265.

Willeboordse, F. H. \& Kaneko, K. [1994] "Bifurcations and spatial chaos in an open flow model," Phys. Rev. Lett. 73(4), 533-536.

Winfree, A. T. [2000] The Geometry of Biological Time (Springer-Verlag, New York), 2nd ed. 
(1)
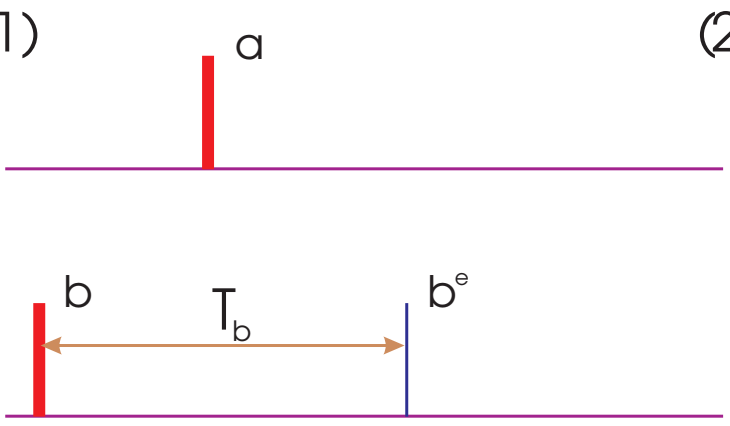

(2)
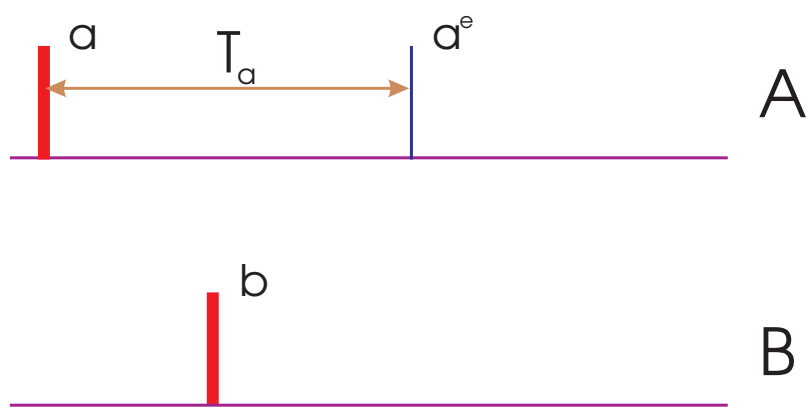

Figure 1: The model of two interacting pulse oscillators. 


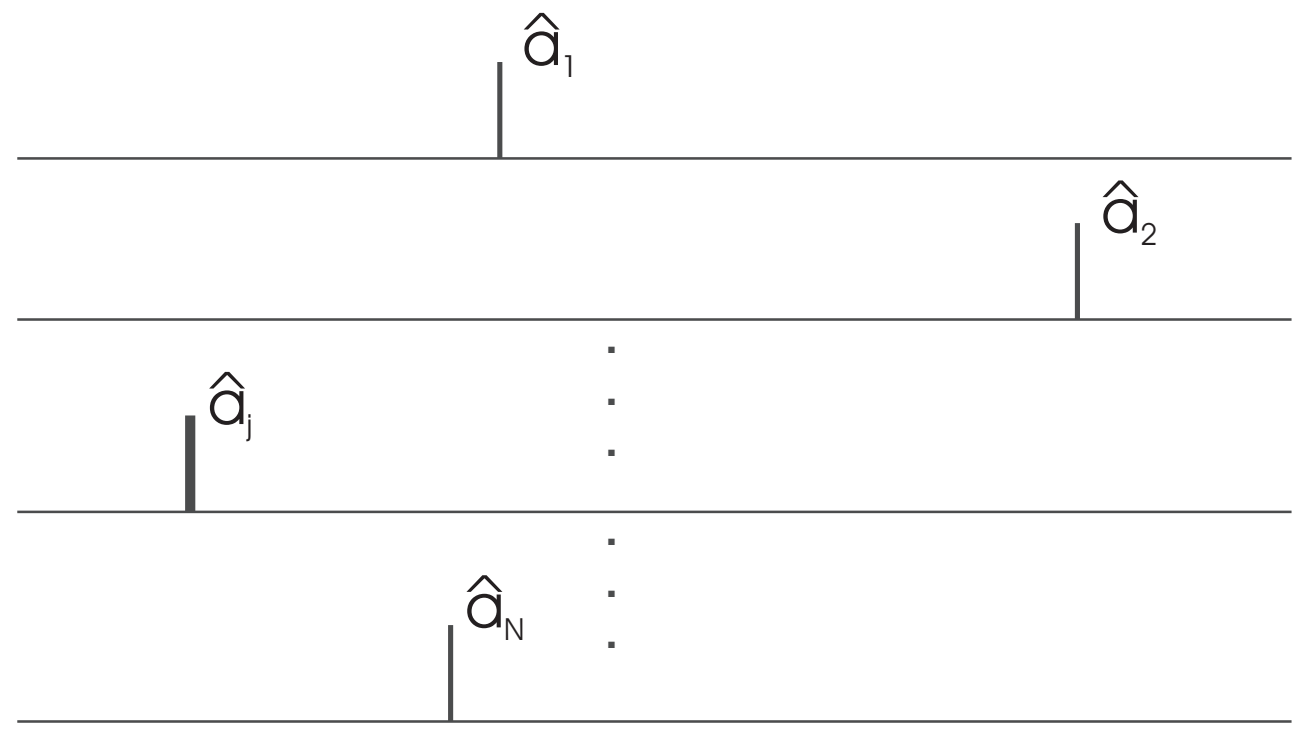

Figure 2: Model of $N$ mutually acting pulse oscillators. 
(a)

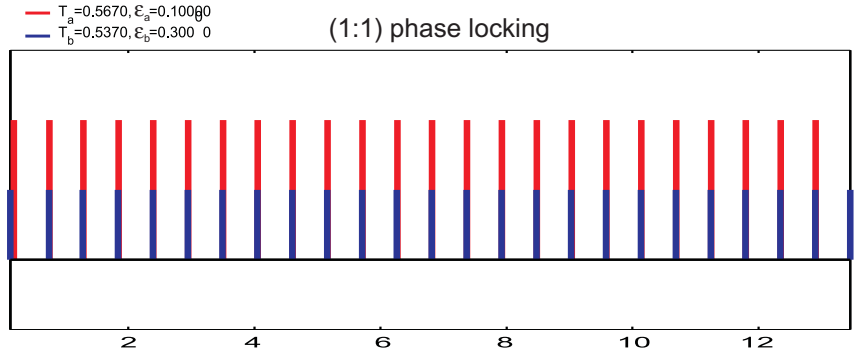

(b) $-\mathrm{T}_{\mathrm{a}}=3.2636, \varepsilon_{\mathrm{a}}=0.05050$

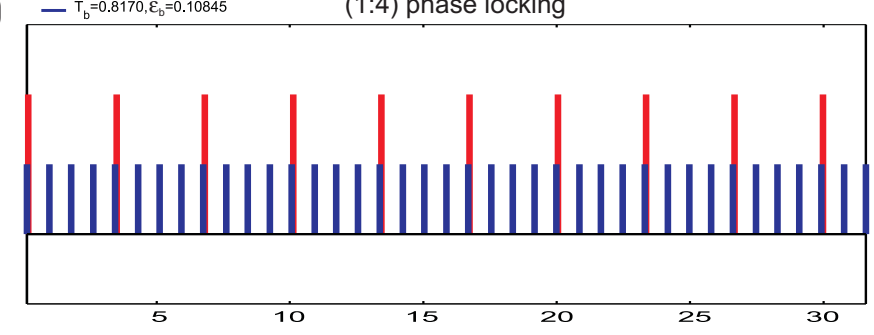

(C) 二 - $\mathrm{T}_{\mathrm{b}}=0.5670, \varepsilon_{\mathrm{a}}=0.6370 . \varepsilon_{0}=0.23350$

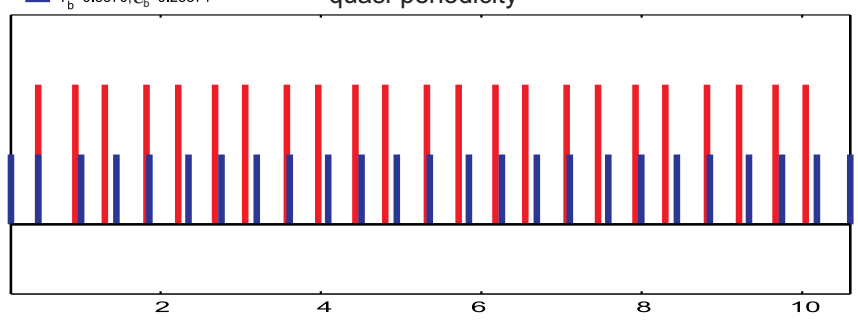

$-\mathrm{T}_{\mathrm{T}}=1.2636 \varepsilon_{\mathrm{a}}=0.12050$

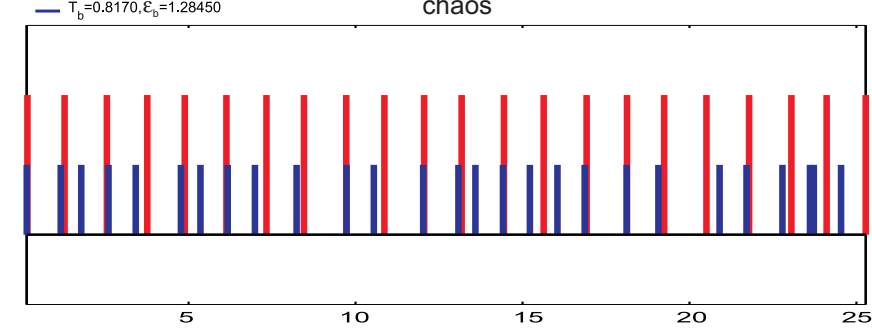

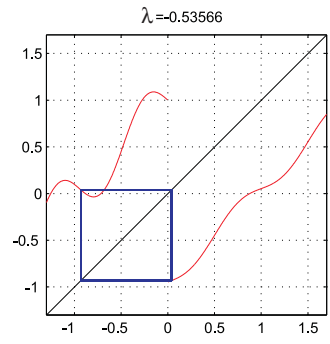
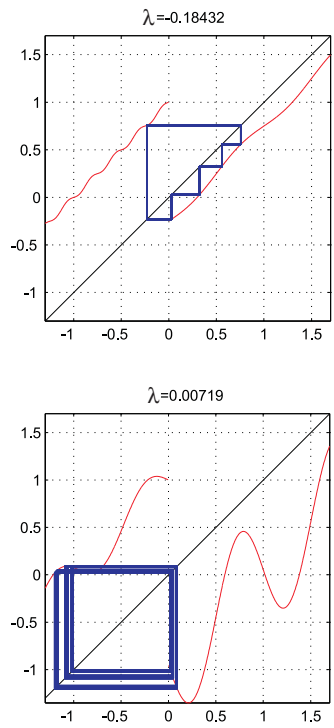

$\lambda=0.59539$

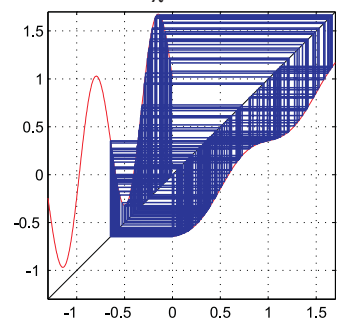

Figure 3: Different types of the behavior of two bidirectionally interacting pacemakers. 
a)

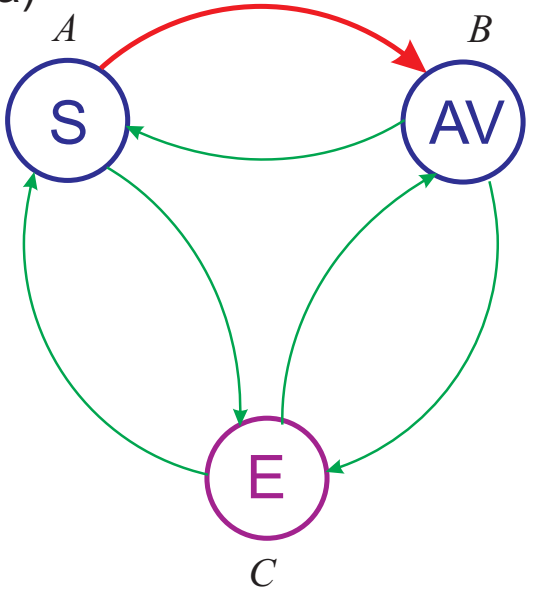

b)

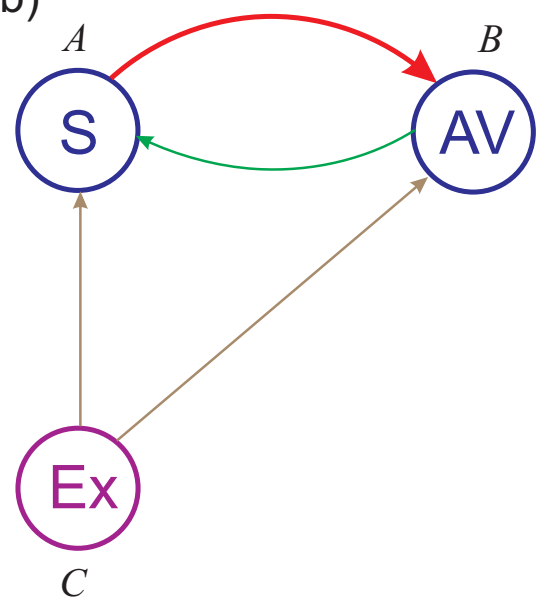

Figure 4: Schematic representation of three pacemakers in the cardiac tissue. 
(a) $-\mathrm{T}_{\mathrm{a}}=1.2630, \varepsilon_{\mathrm{ab}}=0.140, \varepsilon_{\mathrm{ac}}=0.110$ $\begin{aligned} & \mathrm{T}_{\mathrm{b}}=1.4100, \varepsilon_{\mathrm{ba}}=0.220, \varepsilon_{\mathrm{bc}}=0.130 \\ &-\mathrm{T}_{\mathrm{c}}=1.0980, \varepsilon_{\mathrm{ca}}=0.310, \varepsilon_{\mathrm{cb}}=0.120\end{aligned}$

$(1: 1: 1)$ phase locking
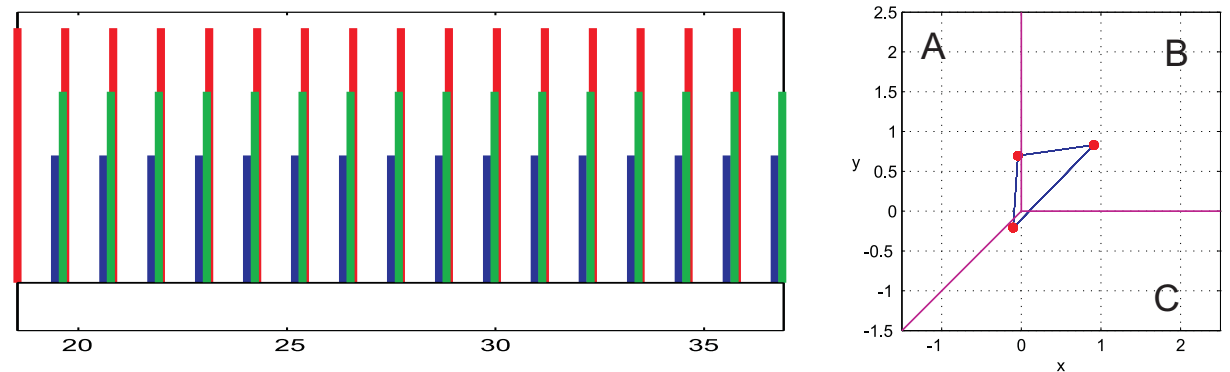

(b) $-\mathrm{T}_{\mathrm{a}}=1.2630, \varepsilon_{\mathrm{ab}}=0.140, \varepsilon_{\mathrm{ac}}=0.110$ $\begin{aligned} & \mathrm{T}_{\mathrm{b}}=0.4210, \varepsilon_{\mathrm{ba}}=0.022, \varepsilon_{\mathrm{bc}}=0.130 \\ &-\mathrm{T}_{\mathrm{c}}=2.0980, \varepsilon_{\mathrm{ca}}=0.310, \varepsilon_{\mathrm{c}}=0.012\end{aligned}$ (2:6:1) phase locking
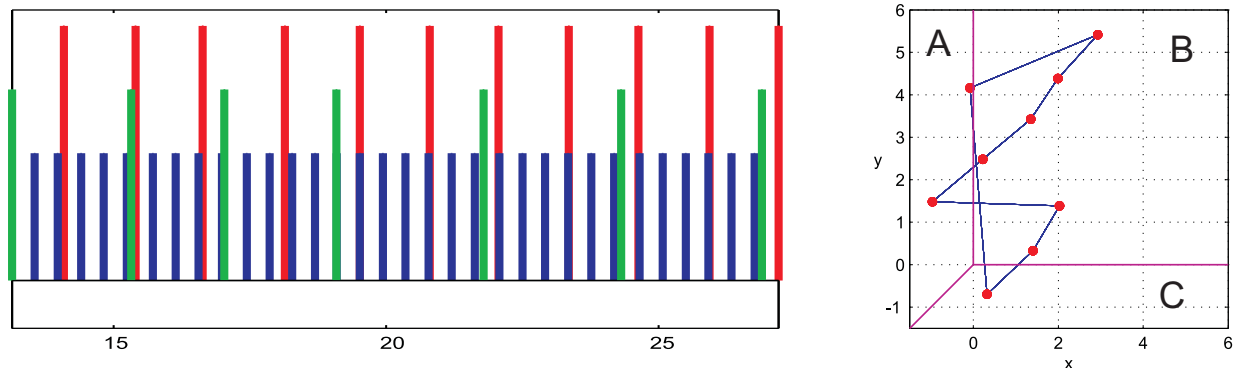

(C) $-\mathrm{T}_{\mathrm{a}}=1.2635, \varepsilon_{\mathrm{ab}}=0.140, \varepsilon_{\mathrm{ac}}=0.110$ - $\mathrm{T}_{\mathrm{b}}=0.4218, \varepsilon_{\mathrm{bba}}=0.420, \varepsilon_{\mathrm{bc}}=0.530 \quad$ complex behavior
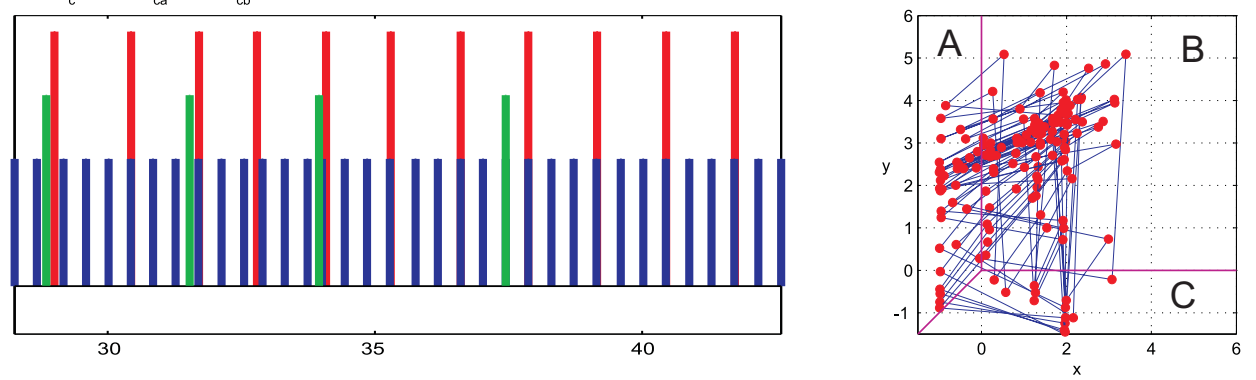

Figure 5: Different types of the behavior of three pulse oscillators. 
a)

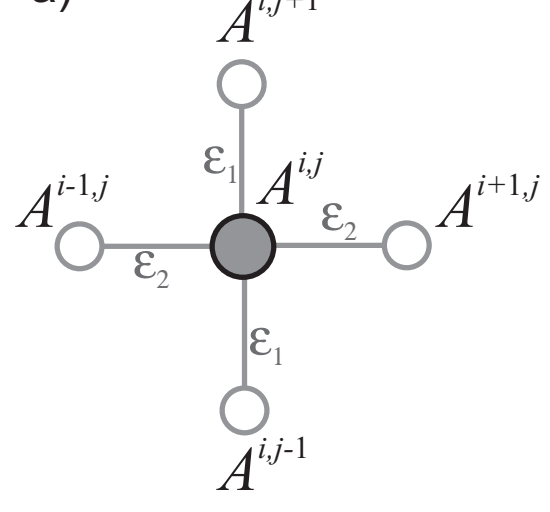

b)

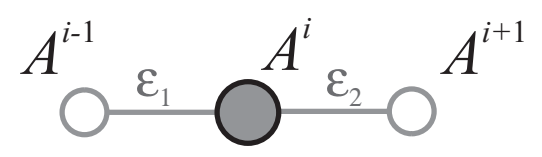

Figure 6: $2 D$ and $1 D$ lattices of coupled pulse oscillators. 